\title{
SUSY VACUA VERSUS CHIRAL FERMIONS
}

\author{
Y. Meurice ${ }^{*}$ ) \\ Institut de Physique Théorique \\ Université Catholique de Louvain \\ Louvain-La-Neuve
}

and

G. Veneziano

CERN - Geneva

\section{A B S T R A C T}

Properties of supersymmetric ground states in a class of fourdimensional theories with chiral fermions are investigated. Constraints on such vacua conflict with a small-size instanton effect which, unless unexpectedly cancelled, spontaneously breaks supersymmetry.

*)

Boursier I.R.S.I.A. 
1. Present understanding of non-perturbative properties of supersymmetric (SUSY) gauge theories in four dimensions rests on a number of approaches:

i) Witten index considerations $\left.{ }^{1-3}\right)$;

ii) effective Lagrangian results $\left.{ }^{4-8}\right)$;

iii) ordinary and anomalous Ward identity arguments ${ }^{9-12)}$;

iv) dynamical calculations using topologically non-trivial fields ${ }^{13-16)}$;

v) anomaly-matching conditions $17-19$ ).

In the case of gauge theories with real fermions, in particular for SUSY-YangMills theory and massive SUSY-QCD (SQCD), the absence of spontaneous SUSY breaking predicted by index calculations $\left.{ }^{1-3}\right)$ has been confirmed $\left.{ }^{4-8}, 13-16\right)$. Furthermore, the phenomenon of SQCD vacua going off to infinity in the massless limit, originally found in the effective Lagrangian approach ${ }^{5}$, has been substantiated by more general arguments $^{9}$ ) and by explicit calculations ${ }^{15-16}$ ). This state of affairs was eventually understood ${ }^{15-16}$ ) as a non-perturbative breaking of the non-renormalization theorem which ensures the all-order perturbative persistence of tree-1evel SUSY vacua. The runaway vacuum can be $\operatorname{seen}^{15-16}$ ) as being due to the presence of $f 1 a t$ directions in the manifold of perturbative vacua and to the vanishing (resp. nonvanishing) of non-perturbative contributions to the energy at infinite (resp. finite) field strength in asymptotically free theories.

The above interpretation of the vacuum structure of SQCD suggests that chiral theories with no flat directions in the tree-level potential may break SUSY spontaneously owirg to non-perturbative effects.

Hints in this direction can already be obtained via effective Lagrangian arguments, as ciscussed by Nilles sometimes ago ${ }^{8}$. He found that in certain chiral theories the F-term in the effective Lagrangian pushes the vacuum to infinity, precisely as i.t does in massless SQCD for fewer flavours than colours. If one wants to reject: this possibility for chiral theories without flat directions, a D-term of the F'eskin type ${ }^{6}$ ) can be invoked to get rid of such a vacuum, and SUSY gets spontaneously broken with a wel1-defined ground state. 
Very recently Affleck et al. ${ }^{7}$ ) gave further arguments for the necessity of such a scenario, by invoking anomaly matching arguments. As is implicit in the authors' discussion, these can be considered at best as good plausibility arguments for SUSY breaking; a straightforward proof is still lacking.

In this note we claim to be able to go a long way towards establishing such proof. We study the properties of SUSY vacua in a class of chiral theories and find that they conflict with a smal1-size instanton effect. Barring an unlikely cancellation, spontaneous SUSY breaking is induced in several cases.

2. The class of theories we consider are generalized SUSY-Georgi-Glashow models with $S U(N)(N \geq 5)$ gauge superfields coupled to the matter superfields:

$$
\begin{aligned}
& \Phi_{i}^{\alpha}, \alpha, \beta=1,2 \ldots N ; i=1,2 \ldots M(N-4) \\
& X_{\alpha \beta}^{a}=-\lambda_{\beta \alpha,}^{a} \quad a=1,2, \ldots M
\end{aligned}
$$

Thus $\Phi$ belongs to the $\underset{\sim}{\bar{N}}$ representation of $S U(N)$, whilst $X$ is a two-index antisymmetric tensor (the 10 for $N=5$ ). The flavour multiplicities, $M(N-4)$ and $M$, axe chosen so to give an anomaly-free theory. The resulting $\beta$-function,

$$
\beta(g)=-\frac{g^{3}}{8 \pi^{2}}[3(N+M)-M N]+O\left(g^{5}\right)
$$

implies asymptotic freedom if $M<3 N /(N-3)$.

The only renormalizable F-term that can be added to the usual gauge interaction is ${ }^{*}$ )

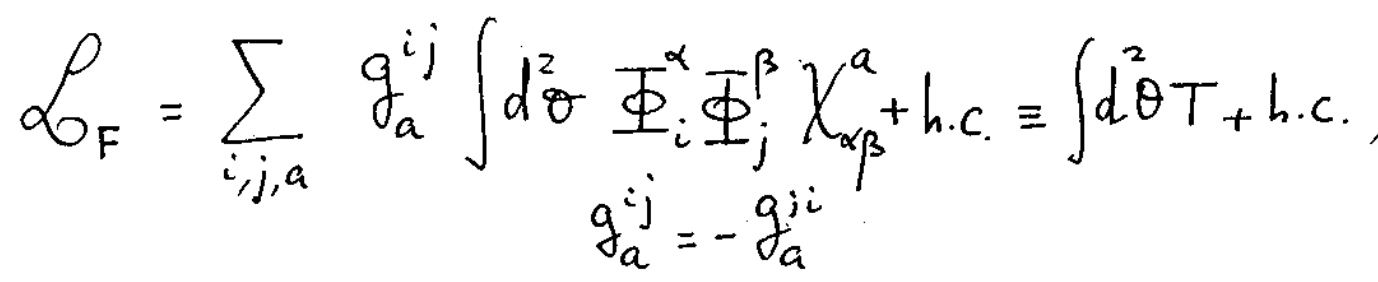

*) For $M=1$ there is no $F$-term; for $N=6$ a term with three $X$ 's can be added. 
For $\mathrm{g}_{\mathrm{a}}^{\mathbf{i j}} \cong 0$ the classical symmetry of this theory is

$$
\sigma_{F}^{l}=S U(M)_{x} \otimes S U(M(N-4))_{\phi} \times U(1)^{3}
$$

The SU factors are anomaly free, commute with SUSY, and act, in the usual way, on the superfields $x^{a}$ and $\Phi_{i}$. The remaining three $U(1)$ factors in Eq. (3) are flavour blind and can be conveniently spanned by the three independent $U(1)$ 's given in the following table.

\section{Table}

The three independent $U(1)$ charges of the various superfield components:

$\phi, \psi$ belong to $\Phi ; \eta, \chi$ belong to $x$

\begin{tabular}{|c|c|c|c|}
\hline Field Charge & $\mathrm{Q}^{(1)}$ & $\mathrm{Q}^{(2)}$ & $\mathrm{Q}^{(3)}$ \\
\hline$\psi$ & $\mathrm{N}-2$ & 1 & $-2(\mathrm{M}+\mathrm{N})$ \\
$\mathrm{N}$ & $\mathrm{N}-2$ & 1 & $\mathrm{MN}-2(\mathrm{M}+\mathrm{N})$ \\
$X$ & $4-\mathrm{N}$ & -2 & $-2 \mathrm{MN}+4(\mathrm{M}+\mathrm{N})$ \\
$\lambda$ & 0 & -2 & $-\mathrm{MN}+4(\mathrm{M}+\mathrm{N})$ \\
\hline
\end{tabular}

These are chosen so that the charges $Q^{(1)}, Q^{(2)}$ (and combinations thereof) commute with SUSY; $Q^{(1)}$ and $Q^{(3)}$ are anomaly free; and $Q^{(2)}, Q^{(3)}$ are not broken by the F-term.

We immediately see a basic difference with respect to theories with real fermions, such as SQCD, where a single current (the vector current) is anomaly free, is unbroken by the F-term, and commutes with SUSY. No such current can be defined in our class of chiral theories.

3. The starting point of our argument makes use of a recently (re) discovered anomaly ${ }^{11)}$. As far as we can see, the anomaly equations can be extended to the case of chiral fermions with only trivial modifications ${ }^{*}$, and read:

*) We have used point-splitting regularization. 


$$
\begin{aligned}
& \frac{1}{4} \bar{D}^{2}\left[(N-2) \sum_{i} \Phi_{i}^{*} e^{V_{\phi}} \Phi_{i}-(N-4) \sum_{a} \chi_{a}^{*} e^{V_{x}} \ell_{a}\right]=N T \\
& \frac{1}{4} \bar{D}^{2}\left[\sum_{i} \Phi_{i}^{*} e^{V_{\phi}} \Phi_{i}-2 \sum_{a} \chi_{a}^{*} e^{V_{x}} \chi_{a}\right]=-M N S
\end{aligned}
$$

where the chiral superfield $T$ is defined in Eq. (2) and, as in Refs. 4 and 5 ,

$$
S=\frac{g^{2}}{32 \pi^{2}} W^{2} ; W=\text { gauge superfield }
$$

In Eqs. (4) and (5) we have taken those linear combinations of the basic anomalies for $\Phi$ and $X$ that project out the explicit breaking $T$ and the anomaly $\mathrm{s}^{*}$. The immediate consequence of Eqs. (4) and (5) is

$$
\langle T\rangle=\left\langle S^{\prime}\right\rangle=0 \text { (in a susy vacuum) }
$$

A similar reasoning can be applied to argue that other chiral superfields must have vanishing SUSY v.e.v.'s. An example of interest is, for $\mathrm{N}=5$,

$$
Y_{i}^{a b} \equiv g_{c}^{i j} \Phi_{j}^{\alpha} X_{\alpha \beta}^{c} X_{\gamma \delta}^{a} X_{\rho \sigma}^{b} \varepsilon^{\beta \gamma \delta \rho \sigma}
$$

The lowest component of $\mathrm{Y}$ (denoted by $\mathrm{y}_{i}^{\mathrm{ab}}$ ) can be obtained from the anticommutator: ${ }^{* *}$ )

$$
\left\{\bar{Q}, \psi_{\beta}^{* i} \eta_{\gamma \delta}^{a} \eta_{\rho \sigma}^{b} \varepsilon^{\beta \gamma \delta \rho \sigma}\right\}=F_{\beta}^{* i} \eta_{\gamma \delta}^{a} \eta_{\rho \sigma}^{b} \varepsilon_{\beta \gamma \delta \rho \sigma}
$$

after use of the equation of motion $\left.{ }^{* * *}\right) F_{\beta}^{* i}=-2 g_{c}^{i j} \phi_{j}^{\alpha} n_{\alpha \beta}^{c}$.

*) In SQCD this is not possible since the anomaly-free combination also gets no F-term contribution. As a result ${ }^{1,12}$, one gets only a relation between $\langle\mathrm{S}\rangle$ and $\langle\mathrm{T}\rangle$.

$* *)(\phi, \psi, F)$ and $(n, \chi, \tilde{F})$ are the components of $\Phi$ and $x$. Lorentz indices are contracted in an obvious way.

***) No extra anomalous piece seems to appear in such an equation. We are grateful to $\mathrm{K}$. Konishi for a discussion on this issue. 
4. In order to see if we arrive at a contradiction regarding the properties of SUSY vacua, we follow the method of Ref. 16. We consider correlation functions that:

i) contain only lowest components of chiral superfields;

ii) satisfy the single-instanton selection rules.

It is easy to see that the above requirements fix uniquely the correlation function to be of the type

$$
C=T\left\langle(\lambda \lambda)^{N-M N+3 M}(\phi)^{M(N-4)}(y)>\right.
$$

where $\lambda$ is the gaugino field, while $\phi$ and $\eta$ are the lowest components of $\Phi$ and $x$. Indices should, of course, be contracted to form a gauge-invariant object which should not be trivially zero and also satisfies all the non-anomalous internal symmetries. One must also evidently have

$$
N-M N+3 M \geqslant 0, \text { i.e. } M \leqslant N /(N-3)
$$

which is one third of the asymptotic freedom limit. Examples of $G$ are the following. For $\mathrm{N}=5, \mathrm{M}=1$,

$$
\sigma_{1}^{5}=\varepsilon^{\alpha \beta \gamma \delta \epsilon} T\left\langle\lambda \lambda\left(x_{1}\right) \lambda \lambda\left(x_{2}\right) \lambda_{\alpha} \lambda_{\tau} \phi^{\tau^{\sigma}} \eta_{\rho \sigma} \eta_{\beta \gamma} \eta_{\delta \epsilon}\left(x_{3}\right)\right\rangle
$$

and for $\mathrm{N}=5, \mathrm{M}=2$,

$$
\sigma_{2}^{5}=T\left\langle\lambda \lambda\left(x_{1}\right) y_{i}^{a b}\left(x_{2}\right) y_{j}^{c d}\left(x_{3}\right) \varepsilon_{i j} \varepsilon_{a c} \varepsilon_{b d}\right\rangle
$$

with $\mathrm{Y}_{i}^{\mathrm{ab}}$ defined by Eq. (8). Note that both theories (if $\mathrm{g}_{a}^{i j} \neq 0, M=2$ ) have no flat directions in their scalar potential.

Al1 these Green functions cannot depend upon the $x_{i}$ 's in a SUSY vacuum ${ }^{13}$ ). Furthermore, as in the examples of Ref. 16 and as explained there, the single instanton action $8 \pi^{2} / g^{2}$ implies a contribution proportional to $\Lambda^{d_{G}}$, where $\Lambda$ is the renormaljzation group invariant scale of the theory and $\mathrm{d}_{\mathrm{G}}$ is the naive dimension of $G$. As a consequence, the single instanton contribution is 


$$
G=\Lambda^{d_{G}} \int d^{4} x_{0} d \rho f\left(x_{0}, \rho, x_{i}\right)
$$

with a dimensionless integral over the instanton size and position which converges*) to a constant precisely as in previous $\operatorname{cases}^{13}{ }^{16}$ ). Unless an unexpected cancellation occurs, one would conclude, by clustering ${ }^{16)}$, that $\lambda \lambda$ and objects like $\langle\lambda \lambda \eta n \eta \phi\rangle(M=1, N=5)$ or $\langle n \eta n \phi\rangle(M=2, N=5)$ cannot be simultaneously zero. For the case $M=1, N=5$ we are unable to prove by the argument leading to Eq. (9) that $\langle\lambda \lambda n \eta n \phi\rangle=0$ since $g_{a}^{i j} \equiv 0$ for $M=1$. The possibility thus remains that $\langle\lambda \lambda \eta n n \phi\rangle=\infty,\langle\lambda \lambda\rangle=0$ is the SUSY vacuum of this theory, although vacua of this type can be discarded using plausibility arguments ${ }^{17}$ ) (i.e. the absence of that directions in the underlying theory).

For the case $M=2, N=5$, and $g_{a}^{i j} \neq 0$, on the other hand, Eq. (9) can be used, and $\langle\lambda \lambda\rangle=\left\langle\mathrm{Y}_{i}^{\mathrm{ab}}\right\rangle=0$ in contradiction with the single instanton contribution to $\mathrm{G}_{2}^{5}$.

If $M>N /(N-3)[M \geq 3$ for $S U(5)]$ we find ourselves in the same situation as that of SQCD with $N<M$, where no Green function could be found ${ }^{16}$ ) that satisfies our requirements $(i)$ and $(i i)$. It is possible that, in such cases, SUSY remains unbroken. This would be consistent with the $N=M=5$ example of supercomplementarity investigated by Taylor ${ }^{18)}$.

In conclusion, we have found that in theories with chiral fermions the presence of several currents which do not fall into a vector/axial vector classification brings about strong constraints on SUSY vacua. These contraints, once coupled to reliable smal1-size instanton effects, lead in certain cases to a contradiction. Even the SQCD way out of a vacuum at infinity appears to be blocked leaving us with the only possibility of a non-supersymmetric ground state.

In the near future further calculations should not fail to provide a complete systematics of the circumstances under which spontaneous SUSY breaking takes place.

*) This straighforward, but lengthy calculation has been carried out in a few cases (D. Amati, G.C. Rossi and G. Veneziano, unpubl.), assuming that the usual functional integration method applies to the case of chiral fermions. 
GV would like to thank D. Amati, J.P. Derendinger, K. Konishi, G.C. Rossi and S. Templeton for interesting discussions. He also acknowledges the kind hospitality enjoyed as a visiting lecturer at Louvain-La-Neuve in November 1983, when this work was begun. Y.M. would like to thank J. Weyers for interesting discussions and encouragement.

After this work was essentially completed we learned that I. Affleck, M. Dine and $N$. Seiberg were reaching similar conclusions by using essentially the same method (N. Seiberg, private communication). 


\section{REFERENCES}

1) E. Witten, Nucl. Phys. B188 (1981) 513 and $\underline{B 202}$ (1982) 253.

2) S. Cecotti and L. Girarde11o, Phys. Lett. 110B (1982) 39.

3) E. Cohen and C. Gomez, Harvard preprint HUPT-83/A007 (1983).

4) G. Veneziano and S. Yankielowicz, Phys. Lett. 113B (1982) 321.

5) T.R. Taylor, G. Veneziano and S. Yankielowicz, Nucl. Phys. B218 (1983) 493.

6) M.E. Peskin, SLAC-PUB 3061 (1983).

7) A.C. Davis, M. Dine and N. Seiberg, Phys. Lett. 125B (1983) 487.

8) H.P. Nilles, Phys. Lett. 129B (1983) 103.

J.M. Gerard and H.P. Nilles, Phys. Lett. 129B (1983) 243.

9) G. Veneziano, Phys. Lett. 124B (1983) 357 and 128B (1983) 199.

G. Shore, CERN-TH.3593 (1983).

10) U. El1wanger, Phys. Lett. 125B (1983) 54.

11) K. Konishi, CERN-TH. 3722 (1983).

See also: T.E. Clark, O. Piguet and K. Sibold, Nucl. Phys. 159B (1979) 1.

S.J. Gates Jr., N.T. Grisaru, M. Roček and W. Siegel, "Superspace", (Benjamin/ Cummings Publ. Comp., New York, 1983), Chapter 6.

12) K. Konishi and K. Shizuya, Univ. Pisa preprint (1984). •

13) V.A. Novikov, M.A. Shifman, A.I. Vainshtein, M.B. Voloshin and V.I. Zakharov, Nuc1. Phys. B229 (1983) 394; V.A. Novikov, M.A. Shifman, A.I. Vainshtein and V.I. Zakharov, Nuc1. Phys. B229 (1983) 381; ibid. 407.

14) E. Cohen and C. Gomez, Phys. Rev. Lett. 52 (1984) 237.

15) I. Affleck, M. Dine and N. Seiberg, Phys. Rev. Lett. 51 (1983) 1026; Dynamical supersymmetry breaking in SQCD, Institute for Advanced Study preprint, November 1983.

16) G.C. Rossi and G. Veneziano, CERN TH-3771 (1983).

17) I. Affleck, M. Dine and N. Seiberg, Dynamical supersymmetry breaking in chiral theories, Institute for Advanced Study preprint, December 1983.

18) T.R. Taylor, Phys. Lett. 125B (1983) 185; and 128B (1983) 403 . 
19) S. Takeshita, Kyushu preprint 82-HE-4 (1982).

J.M. Gerard, J. Govaerts, Y. Meurice and J. Weyers, Phys. Lett. 116B (1982) 29, CERN TH-3562 (1983) and references therein. 